\title{
Quality of Information Aware Incentive Mechanisms for Mobile Crowd Sensing Systems.
}

\author{
Haiming Jin ${ }^{\dagger} \quad$ Lu Su ${ }^{\S} \quad$ Danyang Chen ${ }^{\S} \quad$ Klara Nahrstedt ${ }^{\dagger} \quad$ Jinhui Xu ${ }^{\S}$ \\ †Department of Computer Science, University of Illinois at Urbana-Champaign, IL, USA \\ §Department of Computer Science and Engineering, State University of New York at Buffalo, NY, USA \\ hjin8@illinois.edu, \{lusu, danyangc\}@buffalo.edu, klara@illinois.edu, jinhui@buffalo.edu
}

\begin{abstract}
Recent years have witnessed the emergence of mobile crowd sensing (MCS) systems, which leverage the public crowd equipped with various mobile devices for large scale sensing tasks. In this paper, we study a critical problem in MCS systems, namely, incentivizing user participation. Different from existing work, we incorporate a crucial metric, called users' quality of information (QoI), into our incentive mechanisms for MCS systems. Due to various factors (e.g., sensor quality, noise, etc.) the quality of the sensory data contributed by individual users varies significantly. Obtaining high quality data with little expense is always the ideal of MCS platforms. Technically, we design incentive mechanisms based on reverse combinatorial auctions. We investigate both the singleminded and multi-minded combinatorial auction models. For the former, we design a truthful, individual rational and computationally efficient mechanism that approximately maximizes the social welfare with a guaranteed approximation ratio. For the latter, we design an iterative descending mechanism that achieves close-tooptimal social welfare while satisfying individual rationality and computational efficiency. Through extensive simulations, we validate our theoretical analysis about the close-to-optimal social welfare and fast running time of our mechanisms.
\end{abstract}

\section{Categories and Subject Descriptors}

C.2.1 [Computer-Communication Networks]: Network Architecture and Design

\section{General Terms}

Algorithm, Design, Economics

\section{Keywords}

Crowd Sensing, Incentive Mechanism, Quality of Information

*This work was supported in part by the National Science Foundation under award numbers CNS-1329686, 1329737, 1330142, and 1330491.

${ }^{\dagger}$ The first two authors contribute equally to this paper.

Permission to make digital or hard copies of all or part of this work for personal or classroom use is granted without fee provided that copies are not made or distributed for profit or commercial advantage and that copies bear this notice and the full citation on the first page. Copyrights for components of this work owned by others than ACM must be honored. Abstracting with credit is permitted. To copy otherwise, or republish, to post on servers or to redistribute to lists, requires prior specific permission and/or a fee. Request permissions from permissions@acm.org.

MobiHoc'15, June 22-25, 2015, Hangzhou, China.

Copyright (C) 2015 ACM 978-1-4503-3489-1/15/06 ...\$15.00.

http://dx.doi.org/10.1145/2746285.2746310.

\section{INTRODUCTION}

The ubiquity of human-carried mobile devices (e.g., smartphones, tablets, etc.) with a plethora of on-board and portable sensors (e.g., accelerometer, compass, camera, etc.) has given rise to the emergence of various people-centric mobile crowd sensing (MCS) systems [1-3]. In a typical MCS system, a cloud-based platform aggregates and analyzes the sensory data provided by the public crowd instead of professionals and dedicatedly deployed sensors. The mobile devices of participating users collect and may process in certain level the data before submitting them to the platform.

Such MCS systems hold a wide spectrum of applications including healthcare, ambient environment monitoring, smart transportation, indoor localization, etc. For example, MedWatcher [2] is a US FDA advocated MCS system for post-market medical device surveillance. Participating users upload photos of their medical devices to a cloud-based platform using the MedWatcher mobile application, which help identify visible problems with the devices. The platform aggregates and analyzes the user-provided information, sends reports to the FDA and alerts users about medical device problems. Such a crowdsourcing paradigm enables easier detection of device safety issues and faster propagation of alerts to device users compared to traditional reporting methods such as mail or telephone. Moreover, air quality monitoring [3] is another area where MCS systems obtain their recent popularity. In such systems, crowdsourced air quality data are aggregated from a large number of people using air quality sensors ported to their smartphones, which help estimate the city or district level air quality.

Participating in such crowd sensing tasks is usually a costly procedure for individual users. On one hand, it consumes users' resources, such as computing power, battery and so forth. On the other hand, a considerable portion of sensing tasks require the submission of some types of users' sensitive private information, which causes privacy leakage for participating users. For example, by uploading the photos of their medical devices, users reveal the types of their illnesses. By submitting air quality estimation samples, users usually reveal information about their locations. Therefore, without satisfactory rewards that compensate participating costs, users will be reluctant to carry out the sensing tasks. However, most of the existing MCS systems are based on voluntary user participation or lack effective incentive mechanisms.

Aware of the paramount importance of stimulating user participation, the research community has recently developed some gametheoretic incentive mechanisms for MCS systems [4-18]. However, most of the existing mechanisms fail to incorporate one important aspect, that is users' quality of information (QoI), into their designs. The meaning of QoI varies for different applications. For example, in the aforementioned MedWatcher system [2] QoI refers to the quality (e.g., resolution, contrast, sharpness, etc.) of up- 
loaded photos. Higher quality ones will help the platform better identify visible device problems. In air quality monitoring MCS systems [3], QoI means a user's estimation accuracy of air quality. The QoI of every user could be affected by various factors, including poor sensor quality, noise, lack of sensor calibration and so forth.

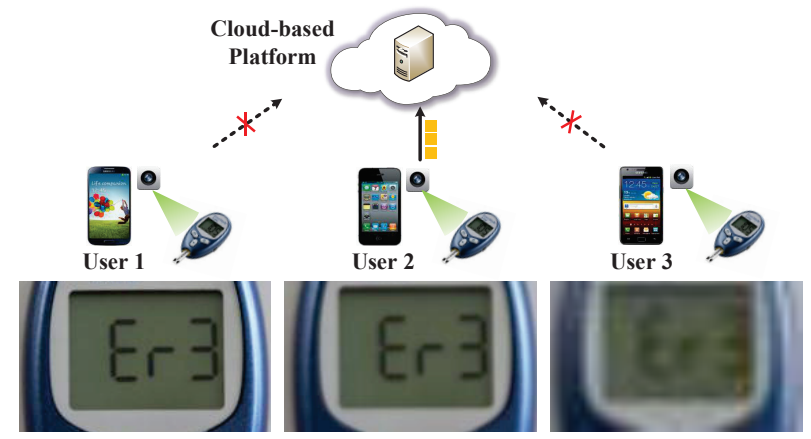

Figure 1: A MedWatcher MCS system example (3 users try to upload the photos of the error message "Er3" on the screens of their blood glucose meters to the MedWatcher platform. The prices that the 3 users ask for cost compensation are $100 \$, 10 \$$ and $1 \$$ respectively.)

To compensate the cost of each user's participation, existing incentive mechanisms have used the user's bidding price as an important metric to allocate sensing tasks. However, as shown in the example in Figure 1, QoI is also a major factor that should be considered together with bidding price. Although user 1 has the highest quality photo, her high price prohibits the platform from requesting her data. Furthermore, despite user 3's low price the platform will not be interested in her data either, because her low quality photo could hardly contribute to identifying the error message "Er3". By jointly considering price and QoI, the platform will select user 2 with medium price and acceptable photo quality as the data provider. Therefore, our goal is to design QoI aware incentive mechanisms for MCS systems.

Considering users' strategic behaviors and the combinatorial nature of the tasks that every user executes, we design incentive mechanisms based on reverse combinatorial auctions, where the platform acts as the auctioneer that purchases the data from participating users. Not only do we study the single-minded scenario where every user is willing to execute one subset of tasks, but also we investigate the multi-minded case in which any user might be interested in executing multiple subsets of tasks. Similar to the traditional VCG mechanisms [19, 20], our mechanisms also aim to maximize the social welfare. Mechanism design for combinatorial auctions is typically challenging in that usually we aim to design a computationally efficient mechanism with close-to-optimal social welfare in the presence of an NP-hard winner determination problem, which meanwhile satisfies truthfulness and individual rationality. Addressing all these challenges, our paper has the following contributions.

- Different from most of the previous work, we design QoI aware incentive mechanisms for MCS systems.

- We use reverse combinatorial auction to design a truthful, individual rational and computationally efficient incentive mechanism that approximately maximizes the social welfare with a guaranteed approximation ratio for the single-minded case.

- For the multi-minded reverse combinatorial auction, we design an iterative descending mechanism that achieves closeto-optimal social welfare with a guaranteed approximation ratio while satisfying individual rationality and computational efficiency.

\section{RELATED WORK}

Game theory has been widely utilized to tackle networking problems such as spectrum sharing [21-25], cooperative communication [26, 27], channel and bandwidth allocation [28, 29] and so forth. Similar to many other problems, when it comes to incentive mechanism design in MCS systems, game theoretic models are also frequently adopted by researchers due to their ability to capture and tackle users' strategic behaviors.

Yang et al. [4] design incentive mechanisms using auction and Stackelberg game for both user-centric and platform-centric models. Their auction-based mechanism, however, does not consider users' untruthful behaviors about bidding task sets. Duan et al. [5] propose a Stackelberg game-based incentive mechanism which is similar to [4] but able to deal with the asymmetrically incomplete information between users and the platform. Faltings et al. [6] design an incentive mechanism to ensure the truthfulness of reported data without considering users' strategic behaviors about bidding prices and task sets. Zhang et al. [30] design an incentive mechanism specifically for binary crowd labelling systems. Furthermore, $[7,8]$ design social cost minimizing incentive mechanisms.

[9-11] study crowdsourcing systems with multiple task requesters and workers. Zhang et al. [9] propose a reputation-based incentive protocol for crowdsourcing applications and use repeated giftgiving game to model the interaction between task requesters and workers. In $[10,11]$, the authors integrate the effort and skill levels of workers into their mechanisms. Another series of work [12-14] utilize online auctions to design incentive mechanisms in crowdsourcing systems where users arrive sequentially.

A common feature of the aforementioned results is that they do not consider QoI in their mechanism designs. This is the major difference with our mechanisms.

In [15], He et al. aim to ensure that every task is executed by enough number of users, which implies that users have identical quality of their sensory data. In contrast, our mechanisms have the ability to handle not only this case, but also the more general case in which users may have heterogeneous QoIs. Authors in [16, 17] design incentive mechanisms based on the assumption of known prior information about the distribution of users' task execution costs. However, we do not assume the availability of such prior knowledge. Furthermore, the QoI aware dynamic participant selection protocol proposed in [18] does not utilize game theoretic frameworks. Hence, it cannot handle users' strategic behaviors as our mechanisms.

\section{PRELIMINARIES}

In this section, we present an overview of MCS systems, our auction model and design objectives.

\subsection{System Overview}

The MCS system model considered in this paper consists of a platform residing in the cloud and a set of $N$ users, denoted as $\mathcal{N}=\{1, \cdots, N\}$. The users execute a set of $M$ sensing tasks, denoted as $\mathcal{T}=\left\{\tau_{1}, \cdots, \tau_{M}\right\}$ and send their sensory data to the platform. The workflow of the system is described as follows.

1. Firstly, the platform announces the set of sensing tasks, $\mathcal{T}$, to users.

2. Then, the platform and users enter the auctioning stage in which the platform acts as the auctioneer that purchases the 
sensory data collected by individual users. Every user $i \in \mathcal{N}$ submits her bid, which is a tuple $\left(\Gamma_{i}, b_{i}\right)$ consisting of the set of tasks $\Gamma_{i} \subseteq \mathcal{T}$ she wants to execute and her bidding price $b_{i}$ for executing these tasks.

3. Based on users' bids, the platform determines the set of winners, denoted as $\mathcal{S} \subseteq \mathcal{N}$ and the payment to all users, denoted as $\vec{p}=\left\{p_{1}, \cdots, p_{N}\right\}$. Specifically, a loser does not execute any task and receives zero payment.

4. After the platform receives winners' sensory data, it gives the payment to the corresponding winners.

One major difference between this paper and most of the previous work is that we integrate the quality of information (QoI) corresponding to every user, denoted as $\vec{q}=\left\{q_{1}, \cdots, q_{N}\right\}$, into our incentive mechanisms. Generally speaking, QoI indicates the quality of users' sensory data. The definition of QoI varies for different applications. For example, in the MedWatcher system [2], QoI refers to the quality (e.g., resolution, contrast, sharpness, etc.) of uploaded photos. Photos with higher quality will help the platform better identify visible problems with medical devices. In air quality monitoring MCS systems [3], QoI refers to a user's estimation accuracy of air quality. We assume that the platform maintains a historical record of users' QoI profile $\vec{q}$ used as inputs for winner and payment determination. There are many methods for the platform to calculate users' QoIs. Intuitively, in the cases where the platform has adequate amount of ground truth data, QoIs can be obtained by directly calculating the deviation of users' data from the ground truths. However, even without ground truths, QoIs can still be effectively inferred from users' data by utilizing algorithms such as those proposed in [31-34]. Alternatively in many applications, QoIs can be inferred from other factors (e.g., the price of a user's sensors, her experience and reputation of executing specific sensing tasks, etc.) using methods proposed in previous studies such as [35]. The problem of which method the platform adopts to calculate users' QoIs is application dependent and out of the scope of this paper. Typically, users might know some of the factors that affect their QoIs. However, users usually do not know exactly how QoIs are calculated by the platform. Hence, they do not know the exact values of their QoIs.

\subsection{Auction Model}

In this paper, we consider strategic and selfish users that aim to maximize their own utilities. The fact that users bid on subsets of tasks motivates us to use reverse combinatorial auction to model the problem. In the rest of the paper, we use bundle to refer to any subset of tasks of $\mathcal{T}$. Different from traditional forward combinatorial auction [36, 37], we formally define the concept of reverse combinatorial auction for our problem setting in Definition 1.

Definition 1 (RC Auction). In a reverse combinatorial auction ( $R C$ auction), each user $i \in \mathcal{N}$ is interested in a set of $K_{i} \geq 1$ bundles, denoted as $\mathcal{T}_{i}=\left\{\Gamma_{i}^{1}, \cdots, \Gamma_{i}^{K_{i}}\right\}$. For any bundle $\Gamma \subseteq \mathcal{T}$, the user has a cost function defined in Equation 1.

$$
C_{i}(\Gamma)= \begin{cases}c_{i}, & \text { if } \exists \Gamma_{i}^{j} \in \mathcal{T}_{i} \text { s.t. } \Gamma \subseteq \Gamma_{i}^{j} . \\ +\infty, & \text { otherwise }\end{cases}
$$

Both $\mathcal{T}_{i}$ and the cost function $C_{i}(\cdot)$ are user $i$ 's private information. If $K_{i}=1$ for every user, then the auction is defined as a single-minded reverse combinatorial auction (SRC auction). And it is defined as a multi-minded reverse combinatorial auction (MRC auction), if $K_{i}>1$ for at least one user.

In an SRC auction, $\mathcal{T}_{i}$ contains only user $i$ 's maximum executable task set $\bar{\Gamma}_{i}$. That is, $\bar{\Gamma}_{i}$ consists of all the sensing tasks that user $i$ is able to execute. Since she is not capable to carry out tasks beyond
$\bar{\Gamma}_{i}$, her cost for any bundle $\Gamma \nsubseteq \bar{\Gamma}_{i}$ can be equivalently viewed as $+\infty$. Similarly in an MRC auction, the union of all the bundles in $\mathcal{T}_{i}$ is $\bar{\Gamma}_{i}$. That is, $\bigcup_{j=1}^{K_{i}} \Gamma_{i}^{j}=\bar{\Gamma}_{i}$. If user $i$ is a winner of the RC auction, she will be paid $p_{i}$ for executing the corresponding set of sensing tasks. In contrast, she will not be allocated any sensing task and will receive zero payment if she is a loser. We present the definitions of the utility of a user and the profit of the platform formally in Definition 2 and 3.

Definition 2 (A User's Utility). The utility of any user $i \in \mathcal{N}$ is

$$
u_{i}= \begin{cases}p_{i}-c_{i}, & \text { if } i \in \mathcal{S} \\ 0, & \text { otherwise }\end{cases}
$$

Definition 3 (Platform's Profit). The profit of the platform given users' QoI profile $\vec{q}$ is

$$
u_{0}=V_{\vec{q}}(\mathcal{S})-\sum_{i \in \mathcal{S}} p_{i},
$$

where the value function $V_{\vec{q}}(\cdot): 2^{\mathcal{N}} \rightarrow \mathbb{R}^{+}$maps the winner set $\mathcal{S}$ to the value that the winners bring to the platform. Furthermore, $V_{\vec{q}}(\cdot)$ is monotonic in $\vec{q}$. That is, for any $\vec{q}=\left\{q_{1}, \cdots, q_{N}\right\}$ and $\vec{q}^{\prime}=\left\{q_{1}^{\prime}, \cdots, q_{N}^{\prime}\right\}$ such that $q_{i} \geq q_{i}^{\prime}$ holds $\forall i \in \mathcal{N}$, we have $V_{\vec{q}}(\mathcal{S}) \geq V_{\vec{q}^{\prime}}(\mathcal{S})$.

Similar to the traditional VCG mechanism design [19, 20], we aim to design mechanisms that maximize the social welfare, which is formally defined in Definition 4.

Definition 4 (Social Welfare). The social welfare of the whole MCS system is

$$
u_{\text {social }}=u_{0}+\sum_{i \in \mathcal{N}} u_{i}=V_{\vec{q}}(\mathcal{S})-\sum_{i \in \mathcal{S}} c_{i} .
$$

\subsection{Design Objective}

In this paper, we aim to design dominant-strategy mechanisms in which for every user there exists a dominant strategy [38] defined in Definition 5.

Definition 5 (Dominant Strategy). A strategy st ${ }_{i}$ is the dominant strategy for user $i$ if and only if for any other strategy $s t_{i}^{\prime}$ and any strategy profile of the other users, denoted as $s t_{-i}$, the property $u_{i}\left(s t_{i}, s t_{-i}\right) \geq u_{i}\left(s t_{i}^{\prime}, s t_{-i}\right)$ holds.

In our SRC auction, each user submits to the platform a bid $\left(\Gamma_{i}, b_{i}\right)$ consisting of her declared interested bundle $\Gamma_{i}$ and the bidding price $b_{i}$. Since users are strategic, it is possible that she declares a bid that deviates from the true value $\left(\bar{\Gamma}_{i}, c_{i}\right)$. However, one of our goals for the SRC auction is to design a truthful mechanism defined in Definition 6.

Definition 6 (Truthfulness). An SRC auction is truthful if and only if it is the dominant strategy for every user $i \in \mathcal{N}$ to bid her true value $\left(\bar{\Gamma}_{i}, c_{i}\right)$.

Noticed from Definition 6 that we aim to ensure the truthfulness of both the cost $c_{i}$ and bundle $\bar{\Gamma}_{i}$. Besides truthfulness, another design objective for the SRC auction is to ensure that every user receives non-negative utility from participating. Such property is critical in incentive mechanisms because it ensures that users will not be disincentivized to participate for receiving negative utilities. This property is defined as individual rationality in Definition 7.

Definition 7 (Individual Rationality). A mechanism is individual rational (IR) if and only if $u_{i} \geq 0$ is satisfied for every user $i \in \mathcal{N}$.

As mentioned in Section 3.2, our mechanism aims to maximize the social welfare. However, as will be proved in Section 4, the problem of maximizing the social welfare in the SRC auction is NP-hard. Hence, we aim to design a polynomial-time mechanism that gives us approximately optimal social welfare with a guaranteed approximation ratio. 


\begin{tabular}{|c|c|c|c|c|c|}
\hline Model & Dominant Strategy & Truthful & IR & Approx. Ratio & Complexity \\
\hline \hline SRC & $\sqrt{ }$ & $\sqrt{ }$ & $\sqrt{ }$ & Guaranteed & Polynomial \\
\hline MRC & $\sqrt{ }$ & $\times$ & $\sqrt{ }$ & Guaranteed & Polynomial \\
\hline
\end{tabular}

Table 1: Summary of design objectives

In the domain of multi-minded combinatorial auction, requiring truthfulness limits the family of mechanisms that can be used, as pointed out in [39]. Hence, in our MRC auction, we aim to design a dominant-strategy mechanism that can still yield a guaranteed approximation ratio to the optimal social welfare without ensuring truthfulness. In fact, as mentioned in [37], the requirement of truthfulness is only to obtain close-to-optimal social welfare with strategic user behaviors, but not the real essence. Therefore, as long as the approximation ratio is guaranteed when users play their dominant strategies, it is justifiable for us to relax the truthfulness requirement. Additionally, we also require our mechanism to be individual rational and have a polynomial computational complexity.

Authors in $[37,40]$ address the issue of mechanism design for multi-minded forward combinatorial auctions. Their mechanisms cannot ensure that users have dominant strategies and cannot be applied to reverse combinatorial auctions. However, in contrast, we are able to design a dominant-strategy incentive mechanism for the MRC auction in this paper. We summarize our design objectives for both the SRC and MRC auctions in Table 1.

\section{SRC AUCTION}

In this section, we introduce the mathematical formulation, mechanism design, an intuitive walk-though example and the corresponding analysis for the SRC auction.

\subsection{Mathematical Formulation}

In our SRC auction, each user's bid $\left(\Gamma_{i}, b_{i}\right)$ consists of her declared interested bundle $\Gamma_{i}$ and the bidding price $b_{i}$. Although our model is valid for any general value function $V_{\vec{q}}(\cdot)$ that satisfies Definition 3, to simplify our analysis we assume that $V_{\vec{q}}(\cdot)$ is the sum of the value, $v_{i}$, contributed by every winner $i \in \mathcal{S}$. Furthermore, we assume that $v_{i}$ is proportional to the total QoI provided by this user. Given users' bidding bundle profile $\vec{\Gamma}=\left\{\Gamma_{1}, \cdots, \Gamma_{N}\right\}$ and the winner set $\mathcal{S}$, the platform's value function $V_{\vec{q}}(\cdot)$ can be represented by Equation 5 .

$$
V_{\vec{q}}(\mathcal{S})=\sum_{i \in \mathcal{S}} v_{i}=\sum_{i \in \mathcal{S}} \alpha q_{i}\left|\Gamma_{i}\right|
$$

where $\alpha$ is a coefficient that transforms QoI to monetary reward.

Another aspect that distinguishes our paper from previous work is that we consider QoI coverage in the SRC auction. For the task that none of the users capable to execute it has adequately high QoI, collective efforts of multiple users are necessary to ensure sensing quality. We use $Q_{\tau_{j}}, \vec{q}(\mathcal{S})$ to denote the total QoI that all winners have on task $\tau_{j} \in \mathcal{T}$. Furthermore, we approximate $Q_{\tau_{j}, \vec{q}}(\mathcal{S})$ as the sum of the QoIs of the winners that execute this task. Therefore, QoI coverage is equivalent to guaranteeing that every task is executed by users with sufficient amount of QoI in total. Based on this additive assumption of QoI, $Q_{\tau_{j}, \vec{q}}(\mathcal{S})$ can be represented by Equation 6.

$$
Q_{\tau_{j}, \vec{q}}(\mathcal{S})=\sum_{i: \tau_{j} \in \Gamma_{i}, i \in \mathcal{S}} q_{i}
$$

Since we aim to maximize the social welfare given in Definition 4 , the winner determination and pricing can be decoupled into two separate problems. We formulate the SRC auction winner determination (SRC-WD) problem as the following integer linear program.

SRC-WD Problem:

$$
\max \sum_{i \in \mathcal{N}}\left(\alpha q_{i}\left|\Gamma_{i}\right|-b_{i}\right) x_{i}
$$

$$
\begin{array}{ll}
\text { s.t. } \sum_{\substack{i: \tau_{j} \in \Gamma_{i}, i \in \mathcal{N} \\
x_{i} \in\{0,1\},}} q_{i} x_{i} \geq Q_{j}, \quad \forall \tau_{j} \in \mathcal{T} \\
& \forall i \in \mathcal{N}
\end{array}
$$

Constants. The SRC-WD problem takes as input constants $\alpha$, users' bid profile $\left\{\left(\Gamma_{1}, b_{1}\right), \cdots,\left(\Gamma_{N}, b_{N}\right)\right\}$, users' QoI profile $\vec{q}$ and tasks' QoI requirement profile $\vec{Q}=\left\{Q_{1}, \cdots, Q_{M}\right\}$.

Variables. In the SRC-WD problem, we have a set of binary variables $\left\{x_{1}, \cdots, x_{N}\right\}$ for every user $i \in \mathcal{N}$. If user $i$ is in the winner set $\mathcal{S}$, then $x_{i}=1$. Otherwise, $x_{i}=0$.

Objective function. Since the platform does not know the true values of users' interested bundles and the corresponding costs, $\left\{\left(\bar{\Gamma}_{1}, c_{1}\right), \cdots,\left(\bar{\Gamma}_{N}, c_{N}\right)\right\}$, the objective function that it directly tries to maximize is the social welfare based on users' bid profile $\left\{\left(\Gamma_{1}, b_{1}\right), \cdots,\left(\Gamma_{N}, b_{N}\right)\right\}$. We use $\vec{w}=\left\{w_{1}, \cdots, w_{N}\right\}$, in which $w_{i}=\alpha q_{i}\left|\Gamma_{i}\right|-b_{i}$, to denote the marginal social welfare profile of all users based on users' bids. Then, we have the objective function $\sum_{i \in \mathcal{S}} w_{i}=\sum_{i \in \mathcal{S}}\left(\alpha q_{i}\left|\Gamma_{i}\right|-b_{i}\right)=\sum_{i \in \mathcal{N}}\left(\alpha q_{i}\left|\Gamma_{i}\right|-\right.$ $\left.b_{i}\right) x_{i}$. Later in Section 4.4, we will show that in our mechanism every user in fact bids truthfully. Hence, the objective function is equivalent to the actual social welfare.

Constraints. Constraint 8 represents the QoI coverage for every task $\tau_{j} \in \mathcal{T}$, which ensures that the total QoI of all the winners for this task, calculated as $Q_{\tau_{j}, \vec{q}}(\mathcal{S})=\sum_{i: \tau_{j} \in \Gamma_{i}, i \in \mathcal{S}} q_{i}=$ $\sum_{i: \tau_{j} \in \Gamma_{i}, i \in \mathcal{N}} q_{i} x_{i}$, is no less than the QoI requirement $Q_{j}$.

Next, we prove the NP-hardness of the SRC-WD problem.

Theorem 1. The SRC-WD problem is NP-hard.

Proof. In this proof, we demonstrate that the NP-complete minimum weight set cover (MWSC) problem is polynomial-time reducible to the SRC-WD problem. The reduction starts with an instance of the MWSC problem consisting of a universe of elements $\mathcal{U}=\left\{\tau_{1}, \cdots, \tau_{M}\right\}$ and a set of $N$ sets $\mathcal{O}=\left\{\Gamma_{1}, \cdots, \Gamma_{N}\right\}$ whose union equals $\mathcal{U}$. Every set $\Gamma_{i} \in \mathcal{O}$ is associated with a non-negative weight $w_{i}$. The MWSC problem is to find the subset of $\mathcal{O}$ with the minimum total weight whose union contains all the elements in $\mathcal{U}$.

Based on the instance of the MWSC problem, we construct an instance of the SRC-WD problem. Firstly, we transform $\Gamma_{i}$ into $\Gamma_{i}^{\prime}$ such that for every element in $\Gamma_{i}$ there exist $l_{i} \in \mathbb{Z}^{+}$copies of the same element in $\Gamma_{i}^{\prime}$. We require that every element $\tau_{j} \in$ $\mathcal{U}$ is covered for at least $L_{j} \in \mathbb{Z}^{+}$times. After the reduction, we obtain an instance of the SRC-WD problem in which users' QoI profile is $\vec{q}=\left\{l_{1}, \cdots, l_{N}\right\}$, users' bidding bundle profile is $\vec{\Gamma}=\left\{\Gamma_{1}, \cdots, \Gamma_{N}\right\}$, users' marginal social welfare profile is $\vec{w}=\left\{-w_{1}, \cdots,-w_{N}\right\}$ and tasks' QoI requirement profile is $\vec{Q}=\left\{L_{1}, \cdots, L_{M}\right\}$. Noticed that the SRC-WD problem represents a richer family of problems in which any user $i$ 's QoI, $q_{i}$, and any task $j$ 's QoI requirement, $Q_{j}$, could take any value in $\mathbb{R}^{+}$. Furthermore, the marginal social welfare can take any value in $\mathbb{R}$. Hence, every instance of the MWSC problem is polynomial-time reducible to an instance of the SRC-WD problem. The SRC-WD problem is NP-hard.

\subsection{Mechanism Design}

Because of the NP-hardness of the SRC-WD problem, it is impossible to compute the set of winners that maximize the social welfare in polynomial time unless $\mathrm{P}=\mathrm{NP}$. As a result, we cannot use the off-the-shelf VCG mechanism $[19,20]$ since the truthfulness of VCG mechanism requires that the social welfare is exactly maximized. Therefore, as mentioned in Section 3.3, we aim to design a mechanism that approximately maximizes the social welfare while guaranteeing truthfulness. 


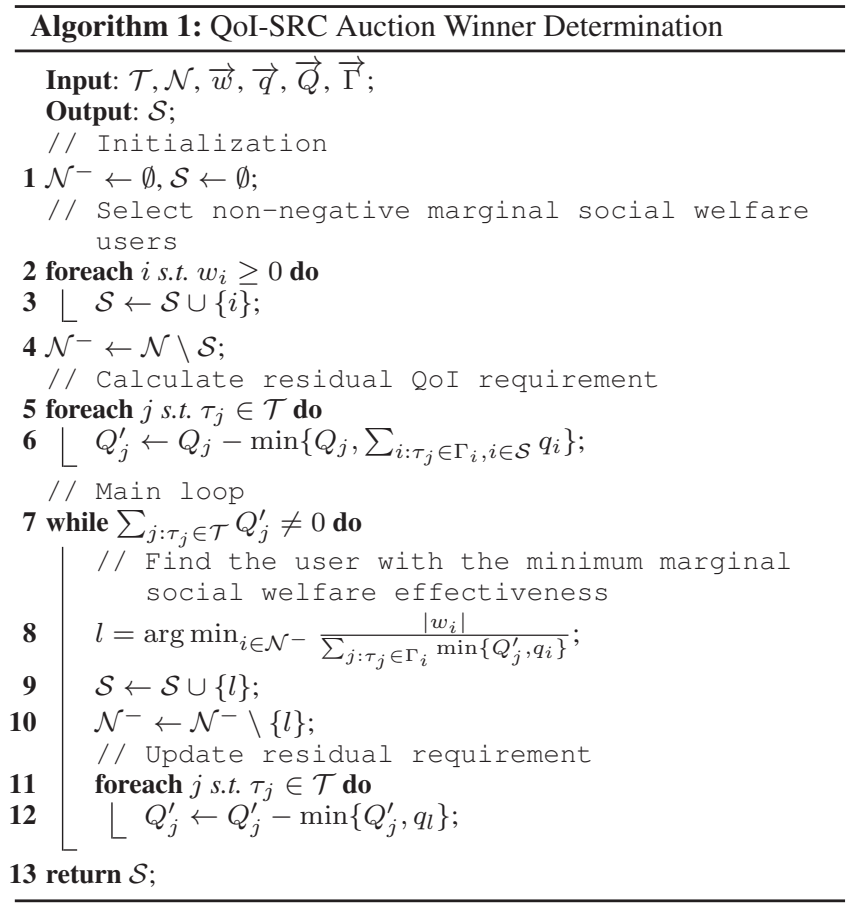

Myerson's characterizations of truthfulness for single-parameter auctions [41] are not directly applicable in our scenario, because our SRC auction is a double-parameter auction that considers both bundle and cost truthfulness. Moreover, different from the characterizations of truthfulness for single-minded forward combinatorial auctions proposed in [36], we describe and prove the necessary and sufficient conditions for a truthful SRC auction in Lemma 1.

Lemma 1. An SRC auction is truthful if and only if the following two properties hold:

- Monotonicity. Any user $i$ who wins by bidding $\left(\Gamma_{i}, b_{i}\right)$ still wins by bidding any $b_{i}^{\prime}<b_{i}$ and any $\Gamma_{i}^{\prime} \supset \Gamma_{i}$ given that other users' bids are fixed.

- Critical payment. Any winner $i$ with bid $\left(\Gamma_{i}, b_{i}\right)$ is paid the supremum of all bidding prices $b_{i}^{\prime}$ such that bidding $\left(\Gamma_{i}, b_{i}^{\prime}\right)$ still wins, which is defined as user i's critical payment.

Proof. It is easily verifiable that a truthful bidder will never receive negative utility. If user $i$ 's any untruthful bid $\left(\Gamma_{i}, b_{i}\right)$ is losing or $\Gamma_{i} \not \subset \bar{\Gamma}_{i}$, her utility from bidding $\left(\Gamma_{i}, b_{i}\right)$ will be non-positive. Therefore, we only need to consider the case in which $\left(\Gamma_{i}, b_{i}\right)$ is winning and $\Gamma_{i} \subseteq \bar{\Gamma}_{i}$.

- Because of the property of monotonicity, $\left(\bar{\Gamma}_{i}, b_{i}\right)$ is also a winning bid. Suppose the payment for bid $\left(\Gamma_{i}, b_{i}\right)$ is $p$ and that for bid $\left(\bar{\Gamma}_{i}, b_{i}\right)$ is $\bar{p}$. Every bid $\left(\bar{\Gamma}_{i}, b_{i}^{\prime}\right)$ with $b_{i}^{\prime}>\bar{p}$ is losing because $\bar{p}$ is the user $i$ 's critical payment given bundle $\bar{\Gamma}_{i}$. From monotonicity, bidding $\left(\Gamma_{i}, b_{i}^{\prime}\right)$ is also losing. Therefore, the critical payment for $\left(\Gamma_{i}, b_{i}\right)$ is at most that for $\left(\bar{\Gamma}_{i}, b_{i}\right)$, which means $p \leq \bar{p}$. Hence, the user will not increase her utility by bidding $\left(\Gamma_{i}, b_{i}\right)$ instead of $\left(\bar{\Gamma}_{i}, b_{i}\right)$.

- Then, we consider the case in which bidding truthfully $\left(\bar{\Gamma}_{i}, c_{i}\right)$ wins. This bid earns the same payment $\bar{p}$ as $\left(\bar{\Gamma}_{i}, b_{i}\right)$. Then her utilities from these two bids will be the same. If bidding $\left(\bar{\Gamma}_{i}, c_{i}\right)$ loses, then we have $c_{i}>\bar{p} \geq b_{i}$. Hence, bidding $\left(\bar{\Gamma}_{i}, b_{i}\right)$ will receive negative utility. Therefore, $\left(\bar{\Gamma}_{i}, b_{i}\right)$ will also not increase her utility compared to $\left(\bar{\Gamma}_{i}, c_{i}\right)$.

Thus, we conclude that an SRC auction is truthful if and only if the monotonicity and critical payment properties hold.

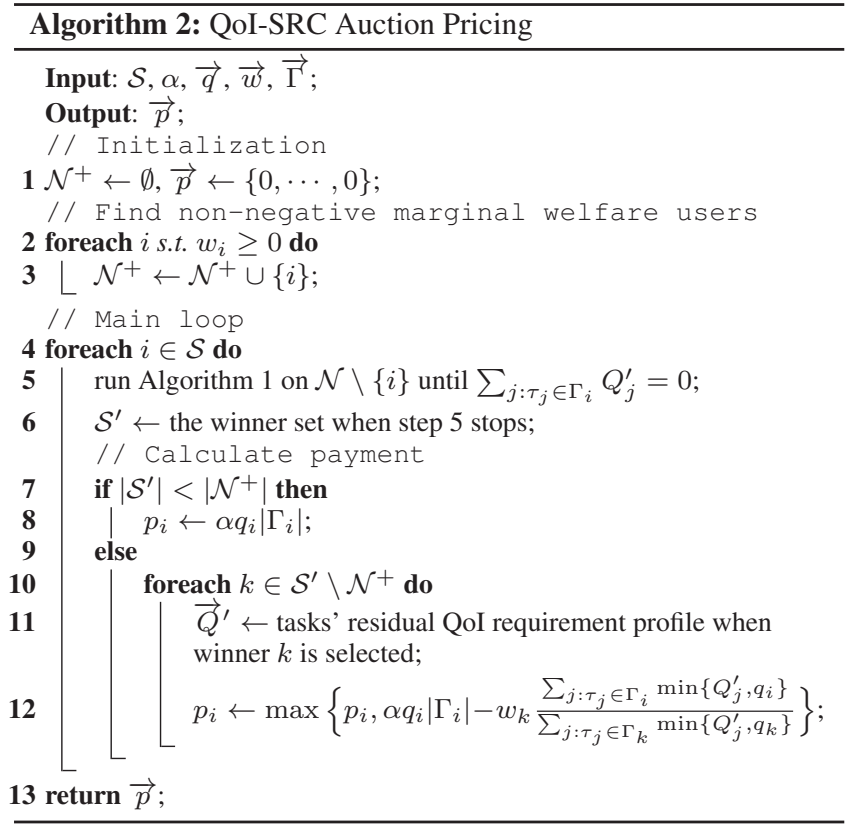

We utilize the rationale provided in Lemma 1 to design a quality of information aware $S R C$ (QoI-SRC) auction. Specifically, we present the winner determination and pricing mechanisms of the QoI-SRC auction respectively in Algorithm 1 and 2.

The platform calculates users' marginal social welfare profile $\vec{w}$ using users' bids $\left\{\left(\Gamma_{1}, b_{1}\right), \cdots,\left(\Gamma_{N}, b_{N}\right)\right\}$ and utilizes $\vec{w}$ as input to the winner determination algorithm shown in Algorithm 1. Firstly, the platform includes all users with non-negative marginal social welfare into the winner set $\mathcal{S}$ (line 2-3). By removing the current winners from $\mathcal{N}$, the platform gets the set of users $\mathcal{N}^{-}$with negative marginal social welfare (line 4). Then, the platform calculates tasks' residual QoI requirement profile $\vec{Q}^{\prime}$ by subtracting from $\vec{Q}$ the QoI provided by the currently selected winners (line 5-6). The main loop (line 7-12) is executed until every task's QoI requirement is satisfied. In the main loop, winner selection is based on marginal social welfare effectiveness (MSWE), defined as the ratio between the absolute value of user $i$ 's marginal social welfare $\left|w_{i}\right|$ and her effective QoI contribution $\sum_{j: \tau_{j} \in \Gamma_{i}} \min \left\{Q_{j}^{\prime}, q_{i}\right\}$. In every iteration, the user with the minimum MSWE among the remaining users in $\mathcal{N}^{-}$is included into $\mathcal{S}$ (line 8-9). After that, the platform updates $\mathcal{N}^{-}$and tasks' residual QoI requirement profile $\vec{Q}^{\prime}$ (line 10-12).

Algorithm 2 describes the corresponding pricing mechanism. It takes the winner set $\mathcal{S}$ as input and outputs the payment profile $\vec{p}$. Firstly, $\vec{p}$ is initialized as a zero vector (line 1 ). Then, the platform includes all users with non-negative marginal social welfare into $\mathcal{N}^{+}$(line 2-3). The main loop (line 4-12) calculates the platform's payment to every winner. For every winner $i \in \mathcal{S}$, the winner determination mechanism in Algorithm 1 is executed with all users except user $i$ until the QoI requirement of every task in $\Gamma_{i}$ has been fully satisfied (line 5). We reach the point such that it is impossible for user $i$ to be selected as a winner in future iterations of Algorithm 1. Then, the platform gets the current winner set $\mathcal{S}^{\prime}$ (line 6) and calculates $p_{i}$ differently in the following two cases.

- Case 1 (line 7-8). Any winner $i$ belonging to case 1 has $w_{i} \geq$ 0 . Hence, this user's critical payment is the bidding price $b_{i}^{\prime}$ that satisfies $w_{i}^{\prime}=\alpha q_{i}\left|\Gamma_{i}\right|-b_{i}^{\prime}=0$. That is, $p_{i}=\alpha q_{i}\left|\Gamma_{i}\right|$.

- Case 2 (line 10-11). For any winner $i$ belonging to case 2, we go through every user $k \in \mathcal{S}^{\prime} \backslash \mathcal{N}^{+}$. We calculate user 
$i$ 's maximum bidding price $b_{i}^{\prime}$ to be able to substitute user $k$ as the winner. That is, $b_{i}^{\prime}$ satisfies Equation 10.

$$
\frac{b_{i}^{\prime}-\alpha q_{i}\left|\Gamma_{i}\right|}{\sum_{j: \tau_{j} \in \Gamma_{i}} \min \left\{Q_{j}^{\prime}, q_{i}\right\}}=\frac{\left|w_{k}\right|}{\sum_{j: \tau_{j} \in \Gamma_{k}} \min \left\{Q_{j}^{\prime}, q_{k}\right\}} .
$$

This means

$$
b_{i}^{\prime}=\alpha q_{i}\left|\Gamma_{i}\right|-w_{k} \frac{\sum_{j: \tau_{j} \in \Gamma_{i}} \min \left\{Q_{j}^{\prime}, q_{i}\right\}}{\sum_{j: \tau_{j} \in \Gamma_{k}} \min \left\{Q_{j}^{\prime}, q_{k}\right\}} .
$$

Finally, the maximum value among all $b_{i}^{\prime}$ 's is used as the payment to user $i$.

\subsection{A Walk-through Example}

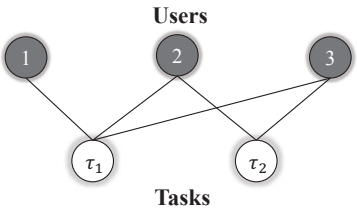

Figure 2: Bidding graph

\begin{tabular}{|c|c|}
\hline Parameters & Value \\
\hline \hline$\alpha$ & 0.1 \\
\hline$\vec{q}$ & $\{0.8,1.2,1.2\}$ \\
\hline$\vec{b}$ & $\{0.2,2.6,2.7\}$ \\
\hline$\vec{Q}$ & $\{1.1,0.8\}$ \\
\hline$\vec{w}$ & $\{0.4,-0.2,-0.3\}$ \\
\hline
\end{tabular}

Table 2: Parameter setting
We use a simple toy example to illustrate how the QoI-SRC auction works. In this example, there are 3 users $\mathcal{N}=\{1,2,3\}$ and 2 tasks $\mathcal{T}=\left\{\tau_{1}, \tau_{2}\right\}$. In Figure 2, an edge between a user $i$ and a task $\tau_{j}$ indicates that $\tau_{j} \in \Gamma_{i}$. That is, users' bidding bundles are $\Gamma_{1}=\left\{\tau_{1}\right\}, \Gamma_{2}=\left\{\tau_{1}, \tau_{2}\right\}$ and $\Gamma_{3}=\left\{\tau_{1}, \tau_{2}\right\}$. Other parameters are shown in Table 2. The process of the QoI-SRC winner determination is shown in Table 3. We show the corresponding pricing process in Table 4

\begin{tabular}{|c|c|c|c|}
\hline $\mathcal{S}$ & $\vec{Q}^{\prime}$ & New Winner & Reason \\
\hline \hline$\emptyset$ & $\{1.1,0.8\}$ & $\{1\}$ & $w_{1}>0$ \\
\hline$\{1\}$ & $\{0.3,0.8\}$ & $\{2\}$ & MSWE $_{\text {user } 2}=\frac{0.2}{1.1}<$ MSWE $_{\text {user } 3}=\frac{0.3}{1.1}$ \\
\hline$\{1,2\}$ & $\{0,0\}$ & $\emptyset$ & $Q_{1}^{\prime}=Q_{2}^{\prime}=0$ and $w_{3}<0$ \\
\hline
\end{tabular}

Table 3: The QoI-SRC auction winner determination

\begin{tabular}{|c|c|c|}
\hline Winner & $\mathcal{S}^{\prime}$ & Payment \\
\hline \hline 1 & $\{2\}$ & $p_{1}=0.1 \times 0.8 \times 1+0.2 \times \frac{0.8}{1.9} \approx 0.164$ \\
\hline 2 & $\{1,3\}$ & $p_{2}=0.1 \times 1.2 \times 2+0.3 \times \frac{1.1}{1.1} \approx 0.540$ \\
\hline
\end{tabular}

Table 4: The QoI-SRC auction pricing

\subsection{Analysis}

Firstly, we analyze the truthfulness and individual rationality of the QoI-SRC auction in Theorem 2 and 3.

Theorem 2. The QoI-SRC auction is truthful.

Proof. Suppose user $i$ wins by bidding $\left(\Gamma_{i}, b_{i}\right)$. We consider user $i$ 's any other bid $\left(\Gamma_{i}^{\prime}, b_{i}^{\prime}\right)$ such that $b_{i}^{\prime}<b_{i}$ or $\Gamma_{i}^{\prime} \supset \Gamma_{i}$.

- Case $1\left(w_{i} \geq 0\right)$. The marginal social welfare for bidding $\left(\Gamma_{i}^{\prime}, b_{i}^{\prime}\right)$ is $w_{i}^{\prime}=\alpha q_{i}\left|\Gamma_{i}^{\prime}\right|-b_{i}^{\prime}>\alpha q_{i}\left|\Gamma_{i}\right|-b_{i} \geq 0$.

- Case $2\left(w_{i}<0\right)$. Bidding $\left(\Gamma_{i}^{\prime}, b_{i}^{\prime}\right)$ will make $w_{i}^{\prime} \geq 0$ or decrease the value of user $i$ 's MSWE.

Hence, user $i$ is still a winner by bidding $\left(\Gamma_{i}^{\prime}, b_{i}^{\prime}\right)$ and the QoISRC auction winner determination algorithm satisfies both bidding bundle and price monotonicity. Furthermore, it is easily verifiable that the pricing mechanism in Algorithm 2 uses the supremum of bidding prices $b_{i}^{\prime}$ such that bidding $\left(\Gamma_{i}, b_{i}^{\prime}\right)$ still wins. Hence, from Lemma 1 we conclude that the QoI-SRC auction is truthful.

Theorem 3. The QoI-SRC auction is individual rational.

Proof. From Theorem 2, we have proved that users bid truthfully in our QoI-SRC auction. Hence, any user $i$ bids its true cost $c_{i}$. Since every winner $i$ is paid the supremum of bidding prices given the bundle $\Gamma_{i}$, we have $p_{i} \geq c_{i}$ for every winner. Apparently, losers have zero utilities in our QoI-SRC auction. Therefore, the utility for every user $i$ satisfies $u_{i} \geq 0$ and the QoI-SRC auction is individual rational.

Then, we analyze the algorithmic properties of the QoI-SRC auction including its computational complexity and approximation ratio to the optimal social welfare in Theorem 4 and 5.

Theorem 4. The computational complexity of the QoI-SRC auction is $O\left(N^{2} M\right)$.

Proof. The computational complexity of Algorithm 1 is dominated by the main loop, which terminates after $N$ iterations in the worst case. In every iteration, the algorithm goes through every task $\tau_{j} \in \mathcal{T}$. Hence, the computational complexity of Algorithm 1 is $O(N M)$. Similarly, we have that the computational complexity of Algorithm 2 is $O\left(N^{2} M\right)$. Therefore, we conclude that computational complexity of the QoI-SRC auction is $O\left(N^{2} M\right)$.

Then, we provide our analysis about the approximation ratio of the QoI-SRC auction using the method similar to the one proposed by Rajagopalan et al. [42]. In our following analysis, we use $\mathcal{N}^{-}$to denote all users $i \in \mathcal{N}$ with negative $w_{i}$ and $\vec{Q}^{-}=$ $\left\{Q_{1}^{-}, \cdots, Q_{M}^{-}\right\}$to denote tasks' residual QoI requirement profile after Algorithm 1 includes all users with $w_{i} \geq 0$ into the winner set. Then, we normalize the $w_{i}$ for every user $i \in \mathcal{N}^{-}$, such that the normalized marginal social welfare $w_{i}^{\prime}=\frac{w_{i}}{\max _{n \in \mathcal{N}^{-}} w_{n}}>0$. Thus, with only a multiplicative factor change to the objective function, we formulate the linear program relaxation of the residual SRC-WD problem defined on user set $\mathcal{N}^{-}$as the normalized primal linear program $\mathbf{P}$. The dual program is formulated in program D.

$$
\begin{array}{cl}
\mathbf{P}: \min \sum_{i \in \mathcal{N}^{-}} w_{i}^{\prime} x_{i} & \\
\text { s.t. } \sum_{\substack{i: \tau_{j} \in \Gamma_{i}, i \in \mathcal{N}^{-} \\
0 \leq x_{i} \leq 1,}} q_{i} x_{i} \geq Q_{j}^{-}, \quad & \forall \tau_{j} \in \mathcal{T} \\
\mathbf{D}: \max \sum_{j: \tau_{j} \in \mathcal{T}} Q_{j}^{-} y_{j}-\sum_{i \in \mathcal{N}^{-}} z_{i} & \\
\text { s.t. } \sum_{j: \tau_{j} \in \Gamma_{i}} q_{i} y_{j}-z_{i} \leq w_{i}^{\prime}, & \forall i \in \mathcal{N}^{-} \\
y_{j} \geq 0, & \forall \tau_{j} \in \mathcal{T}^{-} \\
z_{i} \geq 0, & \forall i \in \mathcal{N}^{-}
\end{array}
$$

It is easily verifiable that the $\left|\max _{i \in \mathcal{N}}-w_{i}\right|$ multiplicative factor difference between the objective functions of $\mathbf{P}$ and the SRCWD problem does not affect the approximation ratio of Algorithm 1. Next, we introduce several notations and concepts utilized in our following analysis.

We define any task $\tau_{j} \in \mathcal{T}$ as alive at any particular iteration of the main loop in Algorithm 1 if its QoI requirement is not fully satisfied. Furthermore, we define that task $\tau_{j}$ is covered by $\Gamma_{i}$ if $\tau_{j} \in \Gamma_{i}$ and $\tau_{j}$ is alive when user $i$ is selected. The coverage relationship is represented as $\tau_{j} \preceq \Gamma_{i}$. Then, we define the minimum measure of QoI as $\Delta q$, the unit QoI. Suppose when user $i$ is about to be selected, the residual QoI requirement profile is $\vec{Q}^{\prime}=\left\{Q_{1}^{\prime}, \cdots, Q_{M}^{\prime}\right\}$ and $\Gamma_{i}$ is the $i_{j}$ th set that covers $\tau_{j}$, the corresponding normalized MSWE in terms of unit QoI can be represented in Equation 19.

$$
W\left(\tau_{j}, i_{j}\right)=\frac{w_{i}^{\prime} \Delta q}{\sum_{j: \tau_{j} \in \Gamma_{i}} \min \left\{Q_{j}^{\prime}, q_{i}\right\}} .
$$

We assume that $\tau_{j}$ is covered by $k_{j}$ sets and we have $W\left(\tau_{j}, 1\right) \leq$ $\cdots \leq W\left(\tau_{j}, k_{j}\right)$ from Equation 19. Then, we define constants $\theta=\max _{i, j} q_{i}\left|\Gamma_{i}\right| w_{j}^{\prime}$ and $m=\frac{1}{\Delta q} \sum_{j: \tau_{j} \in \mathcal{T}} Q_{j}^{-}$that are used in the presentation of the following Lemma 2. 
Lemma 2. The following assignments of $y_{j}$ and $z_{i}$ for $\forall \tau_{j} \in \mathcal{T}$ and $\forall i \in \mathcal{N}^{-}$are feasible to $\boldsymbol{D}$.

$\begin{aligned} y_{j} & =\frac{W\left(\tau_{j}, k_{j}\right)}{2 \theta H_{m} \Delta q}, \forall \tau_{j} \in \mathcal{T}, \\ z_{i} & =\left\{\frac{\sum_{j: \tau_{j} \preceq \Gamma_{i}}\left(\min \left\{Q_{j}^{\prime}, q_{i}\right\}\left(W\left(\tau_{j}, k_{j}\right)-W\left(\tau_{j}, i_{j}\right)\right)\right)}{2 \theta H_{m} \Delta q}, i \in \mathcal{S} .\right. \\ 0, i \notin \mathcal{S} & \end{aligned}$

Proof. Suppose for any user $i \in \mathcal{N}^{-}$, there are $t_{i}$ tasks in bundle $\Gamma_{i}$. We reorder these tasks in the order in which they are fully covered.

If user $i$ is not selected as a winner in $\mathcal{S}$, then we have $z_{i}=0$. Suppose when the last unit QoI of $\tau_{j}$ is about to be covered, the residual QoI requirement profile is $\vec{Q}^{\prime \prime}=\left\{Q_{1}^{\prime \prime}, \cdots, Q_{M}^{\prime \prime}\right\}$, then the total residual QoI of alive tasks contained by $\Gamma_{i}$ is represented as $\sum_{h=j}^{t_{i}} \min \left\{Q_{h}^{\prime \prime}, q_{i}\right\}$. We have

$$
W\left(\tau_{j}, k_{j}\right) \leq \frac{w_{i}^{\prime} \Delta q}{\sum_{h=j}^{t_{i}} \min \left\{Q_{h}^{\prime \prime}, q_{i}\right\}} .
$$

Therefore, we have

$$
\begin{aligned}
\sum_{j=1}^{t_{i}} q_{i} y_{j}-z_{i} & \leq \sum_{j=1}^{t_{i}} \frac{w_{i}^{\prime} q_{i}}{2 \theta H_{m} \sum_{h=j}^{t_{i}} \min \left\{Q_{h}^{\prime \prime}, q_{i}\right\}}-0 \\
& \leq \frac{w_{i}^{\prime}}{H_{m}}\left(1+\frac{1}{2}+\cdots+\frac{1}{m}\right) \\
& \leq w_{i}^{\prime}
\end{aligned}
$$

If user $i \in \mathcal{S}$, then we assume that when user $i$ is selected as a winner, $t_{i}^{\prime}$ tasks in $\Gamma_{i}$ have already been fully covered. We have

$$
\begin{aligned}
& \sum_{j=1}^{t_{i}} q_{i} y_{j}-z_{i} \\
= & \frac{\sum_{j=1}^{t_{i}} q_{i} W\left(\tau_{j}, k_{j}\right)}{2 \theta H_{m} \Delta q}-\frac{\sum_{j=t_{i}^{\prime}+1}^{t_{i}} \min \left\{Q_{j}^{\prime}, q_{i}\right\}\left(W\left(\tau_{j}, k_{j}\right)-W\left(\tau_{j}, i_{j}\right)\right)}{2 \theta H_{m} \Delta q} \\
= & \frac{\sum_{j=1}^{t_{i}^{\prime}} q_{i} W\left(\tau_{j}, k_{j}\right)}{2 \theta H_{m} \Delta q}+\frac{\sum_{j=t_{i}^{\prime}+1}^{t_{i}} \min \left\{Q_{j}^{\prime}, q_{i}\right\} W\left(\tau_{j}, i_{j}\right)}{2 \theta H_{m} \Delta q} \\
& +\frac{\sum_{j=t_{i}^{\prime}+1}^{t_{i}}\left(q_{i}-\min \left\{Q_{j}^{\prime}, q_{i}\right\}\right) W\left(\tau_{j}, k_{j}\right)}{2 \theta H_{m} \Delta q} \\
\leq & \frac{\sum_{j=1}^{t_{i}^{\prime}} \frac{q_{i} w_{i}^{\prime}}{\sum_{h=j}^{t_{i}} \min \left\{Q_{h}^{\prime}, q_{i}\right\}}}{2 \theta H_{m}}+\frac{w_{i}^{\prime}}{2 \theta H_{m}}+\frac{\theta}{2 \theta H_{m}} \\
\leq & w_{i}^{\prime}
\end{aligned}
$$

Therefore, we arrive at the conclusion that the assignments of $y_{j}$ and $z_{i}$ in Lemma 2 are feasible to $\mathbf{D}$.

Then in Theorem 5, we present our result regarding the approximation ratio of Algorithm 1.

Theorem 5. Algorithm 1 is a $2 \theta H_{m}$-approximation algorithm for the residual SRC-WD problem defined on user set $\mathcal{N}^{-}$.

Proof. By substituting the dual assignments given in Lemma 2 into the objective function 15 , we have

$$
\begin{aligned}
& \sum_{j: \tau_{j} \in \mathcal{T}} Q_{j}^{-} y_{j}-\sum_{i \in \mathcal{N}^{-}} z_{i} \\
= & \frac{\sum_{i \in \mathcal{N}^{-} \cap \mathcal{S}} \sum_{j: \tau_{j} \preceq \Gamma_{i}}\left(\min \left\{Q_{j}^{\prime}, q_{i}\right\}\left(W\left(\tau_{j}, i_{j}\right)-W\left(\tau_{j}, k_{j}\right)\right)\right)}{2 \theta H_{m} \Delta q} \\
& +\frac{\sum_{j: \tau_{j} \in \mathcal{T}} Q_{j}^{-} W\left(\tau_{j}, k_{j}\right)}{2 \theta H_{m} \Delta q} \\
= & \frac{\sum_{i \in \mathcal{N}^{-} \cap \mathcal{S}} \sum_{j: \tau_{j} \preceq \Gamma_{i}} \min \left\{Q_{j}^{\prime}, q_{i}\right\} \frac{w_{i}^{\prime} \Delta q}{\sum_{j: \tau_{j} \in \Gamma_{i}} \min \left\{Q_{j}^{\prime}, q_{i}\right\}}}{2 \theta H_{m} \Delta q} \\
= & \frac{\sum_{i \in \mathcal{N}^{-} \cap \mathcal{S}} w_{i}^{\prime}}{2 \theta H_{m}}
\end{aligned}
$$

Because $\mathbf{D}$ is the dual program of $\mathbf{P}$, we have

$$
\frac{\sum_{i \in \mathcal{N}-\cap \mathcal{S}} w_{i}^{\prime}}{2 \theta H_{m}} \leq \mathrm{OPT}_{\mathbf{D}} \leq \mathrm{OPT}_{\mathbf{P}} \leq \mathrm{OPT}_{\mathrm{SRC}-\mathrm{WD}} .
$$

Therefore, Algorithm 1 is a $2 \theta H_{m}$-approximation algorithm for the residual SRC-WD problem defined on user set $\mathcal{N}^{-}$.

\section{MRC AUCTION}

In this section, we present the mathematical formulation, mechanism design and the corresponding analysis for the MRC auction.

\subsection{Mathematical Formulation}

In the MRC auction, we also use the form of the platform's value function $V_{\vec{q}}(\cdot)$ given in Equation 5. If the platform is given users' cost function profile, denote as $\vec{C}=\left\{C_{1}(\cdot), \cdots, C_{N}(\cdot)\right\}$, the MRC auction winner determination (MRC-WD) problem can be formulated as follows.

\section{MRC-WD Problem:}

$$
\begin{array}{cl}
\max \sum_{i \in \mathcal{N}}\left(\alpha q_{i}\left|\Gamma_{i}\right|-C_{i}\left(\Gamma_{i}\right)\right) x_{i} & \\
\text { s.t. } \Gamma_{i} \subseteq \Gamma_{i}^{j}, \exists \Gamma_{i}^{j} \in \mathcal{T}_{i}, & \forall i \in \mathcal{N} \\
x_{i} \in\{0,1\}, & \forall i \in \mathcal{N}
\end{array}
$$

The MRC-WD problem takes the parameter $\alpha$, users' QoI profile $\vec{q}$ and users' cost function profile $\vec{C}$ as input. It has a set of binary variables $\left\{x_{1}, \cdots, x_{n}\right\}$ indicating whether user $i$ is selected in the winner set $\mathcal{S}$. That is, if $i \in \mathcal{S}$, then $x_{i}=1$. Otherwise, $x_{i}=0$.

Furthermore, for every user $i$, we have a variable $\Gamma_{i}$ indicating the set of sensing tasks that the platform allocates to this user. Constraint 21 ensures that $\Gamma_{i}$ is the subset of at least one bundle $\Gamma_{i}^{j} \in \mathcal{T}_{i}$. Therefore, the MRC-WD problem aims to find the set of winners $\mathcal{S}$ and the corresponding task allocation profile denoted as $\vec{\Gamma}=\left\{\Gamma_{1}, \cdots, \Gamma_{N}\right\}$ that maximize the social welfare represented by the objective function. We use $\Gamma_{\max }^{i}$ to denote the bundle with the maximum cardinality in $\mathcal{T}_{i}$ and $w_{\max }^{i}=\alpha q_{i}\left|\Gamma_{\max }^{i}\right|-c_{i}$ to denote user $i$ 's marginal social welfare for $\Gamma_{\max }^{i}$. The maximum social welfare is achieved by selecting all users with positive $w_{\max }^{i}$ as winners and allocating to every winner $i$ the set of tasks $\Gamma_{\max }^{i}$.

However, the challenge is that cost function profile $\vec{C}$ is not known by the platform and we still aim to design a mechanism that approximately maximizes the social welfare with a guaranteed approximation ratio. Then, we present the design of our mechanism in Section 5.2 that achieves this objective while ensuring individual rationality and polynomial computational complexity.

\subsection{Mechanism Design}

Requiring truthfulness in multi-minded combinatorial auctions limits the family of mechanisms that can be used, as mentioned in [39]. As long as the mechanism can achieve close-to-optimal social welfare with a guaranteed approximation ratio, it is justifiable for us to relax the truthfulness requirement, as pointed out in [37]. In Algorithm 3 we describe our design of the iterative descending dominant-strategy quality of information aware MRC (QoI-MRC) auction which is different from the mechanisms designed for multiminded forward combinatorial auctions proposed in [37, 40].

The QoI-MRC auction described in Algorithm 3 consists of a winner determination phase (line 1-18) and a pricing phase (line 19). Every winner $i \in \mathcal{S}$ will be allocated her bidding bundle $\Gamma_{i}$ and be paid her bidding price $b_{i}$ of the final iteration of the winner determination phase. We assume that the platform has the information about the upper bound and lower bound of users' costs denoted as $c_{\max }$ and $c_{\mathrm{min}}$ respectively. The platform initializes every user $i$ 's bidding bundle and bidding price as $\Gamma_{i}=\emptyset$ and $b_{\max } \geq c_{\max }$ (line 2). Moreover, the input parameters $\beta>1$ and $\epsilon \in\left(0, c_{\min }\right]$.

The main loop (line 3-17) is executed until every user is either a winner or a loser. In every iteration of the main loop, every user $i$ such that $\alpha q_{i}\left|\Gamma_{i}\right|-b_{i} \geq \epsilon$ is included in the winner set $\mathcal{S}$ (line 


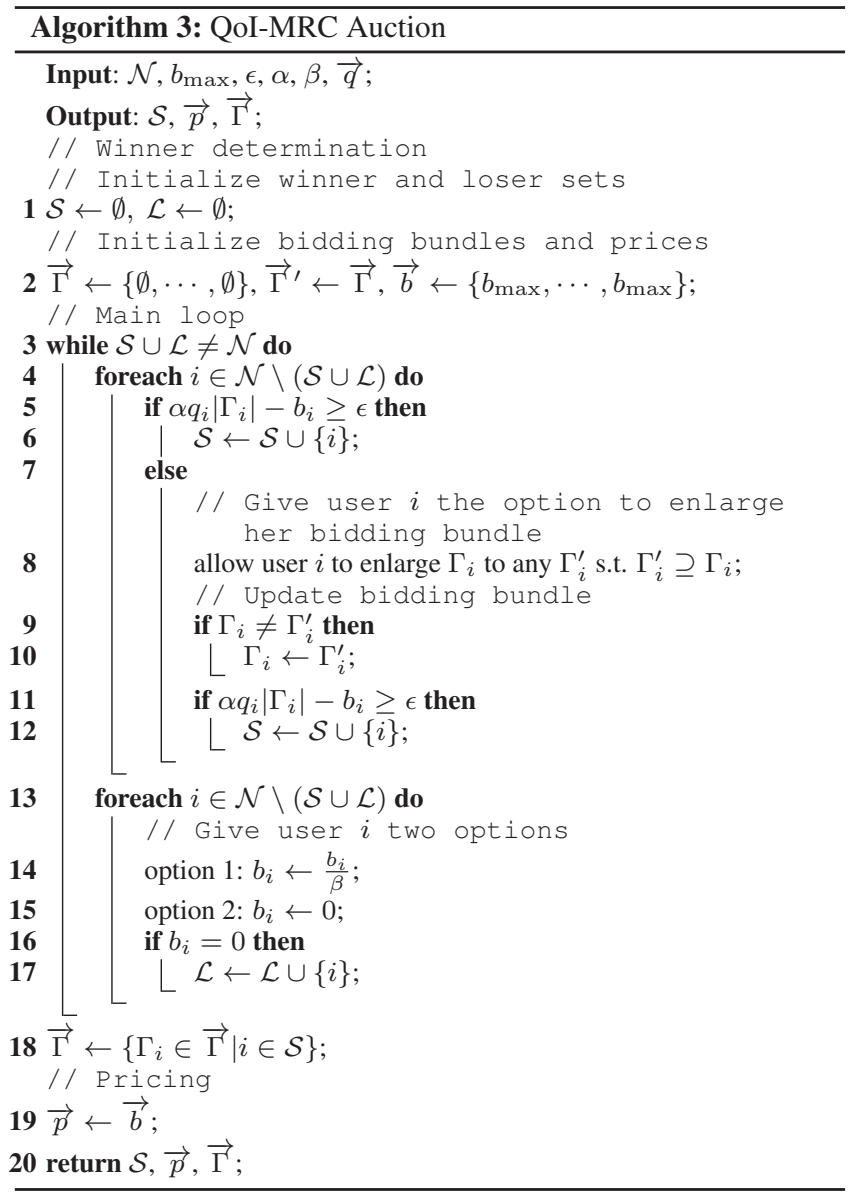

5-6). For any user $i$ that is neither a winner nor a loser in the current iteration, the Algorithm gives her an option to choose whether she will enlarge her current bidding bundle $\Gamma_{i}$ to any bundle $\Gamma_{i}^{\prime}$ that contains $\Gamma_{i}$ (line 5). If after the bundle enlarging $\alpha q_{i}\left|\Gamma_{i}^{\prime}\right|-b_{i} \geq \epsilon$ holds, this user is included in the winner set (line 11-12). Otherwise, she is given the following two options to choose from.

- Option 1 (line 14). By choosing option 1, user $i$ divides her bidding price $b_{i}$ by $\beta$. As long as she is fully rational, she will choose option 1 rather than option 2 to drop out of the auction, if $\frac{b_{i}}{\beta}>c_{i}$ hold. By doing so, she keeps herself in the auction and makes it still possible for her to win in one of the future iterations to receive positive utility.

- Option 2 (line 15). By choosing option 2, the user $i$ drops out of the auction. If $\frac{b_{i}}{\beta} \leq c_{i}$, any rational user $i$ will choose option 2 because it is impossible for her to obtain positive utility even though she remains in the auction in this case.

Finally, every winner $i$ is allocated her bidding bundle $\Gamma_{i}$ (line 18) and be paid her bidding price $b_{i}$ (line 19) of the final iteration of the winner determination phase.

\subsection{Analysis}

Although the QoI-MRC auction cannot guarantee truthfulness because users' bidding prices when Algorithm 3 terminates will possibly not be equal to users' true costs, we show in the following Theorem 6 that every user still has a dominant strategy.

Theorem 6. Every user $i \in \mathcal{N}$ has the following dominant strategy in the QoI-MRC auction.

- User i enlarges bundle $\Gamma_{i}$ to $\Gamma_{\max }^{i}$ in the first iteration.
- When user $i$ is given the options to divide her bidding price $b_{i}$ by $\beta$ or drop out of the auction, she will always choose the former as long as $\frac{b_{i}}{\beta}>c_{i}$ and the latter if $\frac{b_{i}}{\beta} \leq c_{i}$.

Proof. Obviously, any rational user $i$ will choose to divide her current bidding price $b_{i}$ by $\beta$ as long as $\frac{b_{i}}{\beta}>c_{i}$ when she is given the two options. By doing so, it is still possible for her to win the auction and be paid $p_{i}>c_{i}$. If $\frac{b_{i}}{\beta} \leq c_{i}$, then even if she wins the auction the payment $p_{i}$ will not be larger than $c_{i}$. Hence, she will drop out in this case.

Then, we study whether any user $i$ will enlarge her bundle to some $\Gamma_{i}^{\prime} \neq \Gamma_{\max }^{i}$ in the first iteration.

- Case $1\left(\alpha q_{i}\left|\Gamma_{\max }^{i}\right|-b_{\max }>\alpha q_{i}\left|\Gamma_{i}^{\prime}\right|-b_{\max } \geq \epsilon\right)$. Both $\Gamma_{\max }^{i}$ and $\Gamma_{i}^{\prime}$ will make the user win the auction in the first iteration and be paid $b_{\max }$. We have $u\left(\Gamma_{\max }^{i}\right)=u\left(\Gamma_{i}^{\prime}\right)$.

- Case $2\left(\alpha q_{i}\left|\Gamma_{\max }^{i}\right|-b_{\max } \geq \epsilon>\alpha q_{i}\left|\Gamma_{i}^{\prime}\right|-b_{\max }\right)$. The user will win and be paid $b_{\max }$ by enlarging to $\Gamma_{\max }^{i}$ in the first iteration and we have $u\left(\Gamma_{\max }^{i}\right)=b_{\max }-c_{i}$. If she proposes $\Gamma_{i}^{\prime}$ instead of $\Gamma_{\max }^{i}$, she will be asked to decrease her bid or drop out in the first iteration. Eventually, she could lose or win with being paid $b_{i}^{\prime}<b_{\max }$. Her utility could either be $u\left(\Gamma_{i}^{\prime}\right)=0$ or $u\left(\Gamma_{i}^{\prime}\right)=b_{i}^{\prime}-c_{i}$. We have $u\left(\Gamma_{\max }^{i}\right)>u\left(\Gamma_{i}^{\prime}\right)$.

- Case 3 ( $\left.\epsilon>\alpha q_{i}\left|\Gamma_{\max }^{i}\right|-b_{\max }>\alpha q_{i}\left|\Gamma_{i}^{\prime}\right|-b_{\max }\right)$. Both $\Gamma_{\max }^{i}$ and $\Gamma_{i}^{\prime}$ will make the user face the choices of decreasing her bid or dropping out in the first iteration. If eventually she wins in both cases, then the number of iterations before she wins if she proposes $\Gamma_{\max }^{i}$ will be smaller than or equal to that of $\Gamma_{i}^{\prime}$. The payments $p_{i}$ and $p_{i}^{\prime}$ for the two cases satisfy $p_{i} \geq p_{i}^{\prime}$ and we have $u\left(\Gamma_{\max }^{i}\right) \geq u\left(\Gamma_{i}^{\prime}\right)$. If she loses in both cases, then $u\left(\Gamma_{\max }^{i}\right)=u\left(\Gamma_{i}^{\prime}\right)=0$. The last scenario is that she wins by proposing $\Gamma_{\max }^{i}$ and loses by proposing $\Gamma_{i}^{\prime}$ in the first iteration. Then, we have $u\left(\Gamma_{\max }^{i}\right)>0=u\left(\Gamma_{i}^{\prime}\right)$.

We have $u\left(\Gamma_{\max }^{i}\right) \geq u\left(\Gamma_{i}^{\prime}\right)$ with at least one scenario with strict inequality. Hence, user $i$ enlarges bundle $\Gamma_{i}$ to $\Gamma_{\max }^{i}$ in the first iteration. We arrive at the conclusion about any user's dominant strategy stated in Theorem 6.

Theorem 7. The QoI-MRC auction is individual rational.

Proof. When a user is given the choices to decrease her bid or drops out of the auction, any user $i$ will drop out if $\frac{b_{i}}{\beta} \leq c_{i}$. She becomes a loser and obtains $u_{i}=0$. The user only chooses to divide $b_{i}$ by $\beta$ if $\frac{b_{i}}{\beta}>c_{i}$, which ensures that her payment $p_{i}>c_{i}$ if she wins. In this case, we have $u_{i}>0$. Therefore, $u_{i} \geq 0$ and the QoI-MRC auction is individual rational.

Then, we analyze the algorithmic properties of the QoI-MRC auction including its computational complexity and approximation ratio in Theorem 8 and 9.

Theorem 8. The computational complexity of the QoI-MRC auction is $O(N)$.

Proof. It is easily verifiable that the main loop of Algorithm 3 terminates after $O\left(\log _{\beta} \frac{b_{\max }}{c_{\min }}\right)$ number of iterations. The computational complexity inside the main loop is $O(N)$. Therefore, the computational complexity of the QoI-MRC auction is $O(N)$.

In Theorem 9, we present our results about the approximation ratio of the QoI-MRC auction to the optimal social welfare. We use $\mathcal{S}_{\text {OPT }}$ to denote the winner set of the optimal solution of the MRCWD problem, $q_{\max }$ to denote the maximum QoI in $\vec{q}$ and $\Gamma_{\max }$ to denote the maximum-cardinality bundle in $\left\{\Gamma_{\max }^{1}, \cdots, \Gamma_{\max }^{N}\right\}$.

Theorem 9. The approximation ratio of the QoI-MRC auction to the optimal social welfare is $\frac{|\mathcal{S}| \epsilon}{\left|\mathcal{S}_{O P T}\right|\left(\alpha q_{\max }\left|\Gamma_{\max }\right|-c_{\min }\right)}$.

Proof. We use APP to denote the social welfare resulted by the QoI-MRC auction. From Theorem 6, every user $i \in \mathcal{N}$ enlarges her bundle to $\Gamma_{\max }^{i}$ in the first iteration. The winner set $\mathcal{S}$ output 
by Algorithm 3 consists of winners $\mathcal{S}_{1}$ that win in the first iteration and $\mathcal{S}_{2}$ that win in iteration $r_{i}>1$ with bidding price $b_{i}^{r_{i}}$. We have

$$
\begin{aligned}
\operatorname{APP} & =\sum_{i \in \mathcal{S}}\left(\alpha q_{i}\left|\Gamma_{\max }^{i}\right|-c_{i}\right) \\
& \geq \sum_{i \in \mathcal{S}_{1}}\left(\alpha q_{i}\left|\Gamma_{\max }^{i}\right|-b_{\max }\right)+\sum_{i \in \mathcal{S}_{2}}\left(\alpha q_{i}\left|\Gamma_{\max }^{i}\right|-b_{i}^{r_{i}}\right) \\
& \geq\left|\mathcal{S}_{1}\right| \epsilon+\left|\mathcal{S}_{2}\right| \epsilon \\
& =|\mathcal{S}| \epsilon
\end{aligned}
$$

Similarly, the optimal solution OPT is

$$
\begin{aligned}
\mathrm{OPT} & =\sum_{i \in \mathcal{S}_{\mathrm{OPT}}}\left(\alpha q_{i}\left|\Gamma_{\text {max }}^{i}\right|-c_{i}\right) \\
& \leq\left|\mathcal{S}_{\mathrm{OPT}}\right|\left(\alpha q_{\max }\left|\Gamma_{\max }\right|-c_{\text {min }}\right) \\
& =\frac{\left|\mathcal{S}_{\mathrm{OPT}}\right|\left(\alpha q_{\max }\left|\Gamma_{\max }\right|-c_{\min }\right)}{|\mathcal{S}| \epsilon} \cdot|\mathcal{S}| \epsilon \\
& \leq \frac{\left|\mathcal{S}_{\mathrm{OPT}}\right|\left(\alpha q_{\max }\left|\Gamma_{\max }\right|-c_{\min }\right)}{|\mathcal{S}| \epsilon} \cdot \mathrm{APP}
\end{aligned}
$$

Therefore, the approximation ratio of the QoI-MRC auction to the optimal social welfare is $\frac{|\mathcal{S}| \epsilon}{\left|\mathcal{S}_{\text {OPT }}\right|\left(\alpha q_{\max }\left|\Gamma_{\max }\right|-c_{\min }\right)}$.

\section{PERFORMANCE EVALUATION}

\subsection{Baseline Method}

The first baseline approach is a modified version of the traditional VCG auction $[19,20]$. We integrate the concept of QoI and the QoI coverage constraint defined in Section 4 into the VCG winner determination (VCG-WD) problem. We call the modified VCG auction quality of information aware VCG (QoI-VCG) auction, in which the VCG-WD problem is solved optimally and the VCG pricing mechanism $[19,20]$ is utilized to derive winners' payments.

Another baseline method is the marginal social welfare greedy (MSW-Greedy) auction. Its winner determination algorithm firstly includes every user $i$ with $w_{i} \geq 0$ into the winner set. Then, it selects the user with the largest marginal social welfare among the remaining users in every iteration until tasks' QoI requirements are fully satisfied. The pricing mechanism is similar to Algorithm 2 which essentially pays every winner her supremum bidding price to win given her current bidding bundle. It is easily verifiable that the MSW-Greedy auction is truthful and individual rational.

\subsection{Simulation Settings}

\begin{tabular}{|c|c|c|c|c|c|c|c|}
\hline Setting & $\alpha$ & $c_{i}$ & $q_{i}$ & $Q_{j}$ & $\left|\bar{\Gamma}_{i}\right|$ & $N$ & $M$ \\
\hline \hline I & 0.1 & {$[2,4]$} & {$[1,2]$} & {$[10,13]$} & {$[20,30]$} & {$[200,500]$} & 100 \\
\hline II & 0.1 & {$[4,8]$} & {$[2,4]$} & {$[10,13]$} & {$[20,30]$} & 300 & {$[300,600]$} \\
\hline
\end{tabular}

Table 5: Simulation settings for SRC auction

\begin{tabular}{|c|c|c|c|c|c|c|c|}
\hline Setting & $\alpha$ & $b_{\max }$ & $c_{i}$ & $q_{i}$ & $\left|\bar{\Gamma}_{i}\right|$ & $N$ & $M$ \\
\hline \hline III & 0.2 & 100 & {$[4,6]$} & {$[1,2]$} & {$[20,30]$} & {$[200,500]$} & 100 \\
\hline IV & 0.2 & 100 & {$[6,10]$} & {$[2,4]$} & {$[20,30]$} & 300 & {$[200,400]$} \\
\hline
\end{tabular}

Table 6: Simulation settings for MRC auction

For our simulation of the SRC auction, we consider the two settings described in Table 5. In setting I, we fix the number of tasks as $M=100$ and vary the number of users from 200 to 500 . In setting II, we fix the number of users as $N=300$ and vary the number of tasks from 300 to 600 . The parameter $\alpha=0.1$ in both settings and the values of $c_{i}, q_{i},\left|\bar{\Gamma}_{i}\right|$ for any user $i \in \mathcal{N}$ and $Q_{j}$ for any task $\tau_{j} \in \mathcal{T}$ are generated uniformly at random from the ranges given in Table 5. User $i$ 's maximum executable task set $\bar{\Gamma}_{i}$ consists of $\left|\bar{\Gamma}_{i}\right|$ tasks selected uniformly at random from $\mathcal{T}$. Furthermore, the optimal solution to the VCG-WD problem of the QoI-VCG mechanism is calculated using the GUROBI optimization solver [43].
For our simulation of the MRC auction, we consider the two settings described in Table 6. In setting III, we fix the number of tasks as $M=100$ and vary the number of users from 200 to 500 . In setting II, we fix the number of users as $N=300$ and vary the number of tasks from 200 to 400 . The parameters $\alpha=0.2$ and $b_{\max }=0.2$ in both settings and the values of $c_{i}, q_{i},\left|\bar{\Gamma}_{i}\right|$ for any user $i \in \mathcal{N}$ are generated uniformly at random from the ranges given in Table 6. User $i$ 's maximum executable task set $\bar{\Gamma}_{i}$ consists of $\left|\bar{\Gamma}_{i}\right|$ tasks selected uniformly at random from $\mathcal{T}$. User $i$ 's interested bundle set consists of randomly selected subsets of $\bar{\Gamma}_{i}$ whose union is $\bar{\Gamma}_{i}$.
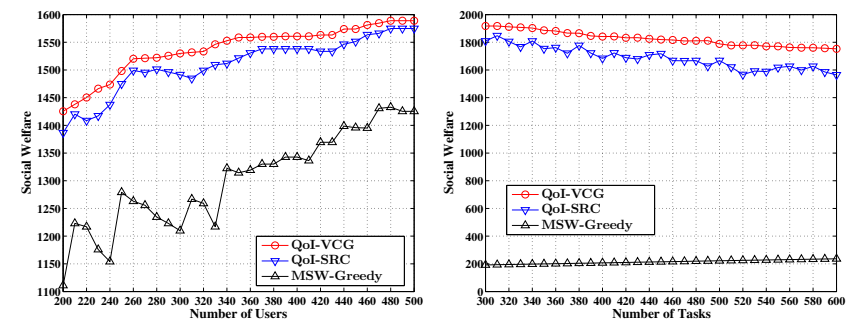

Figure 3: Social welfare for setting I

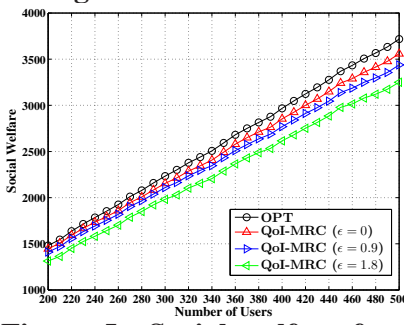

Figure 5: Social welfare for setting III (varying $\epsilon$ )

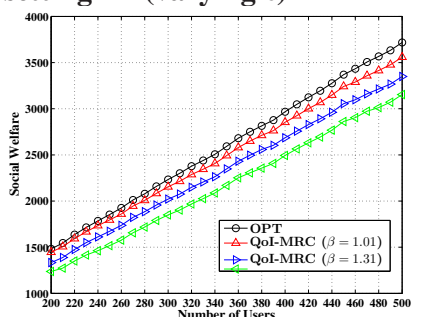

Figure 7: Social welfare for setting III (varying $\beta$ )

Figure 4: Social welfare for setting II

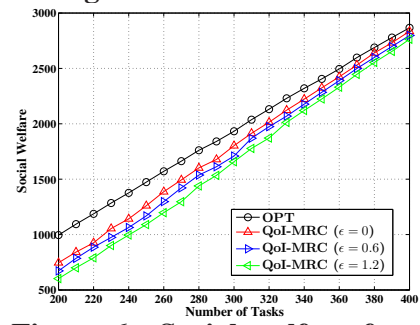

Figure 6: Social welfare for setting IV (varying $\epsilon$ )

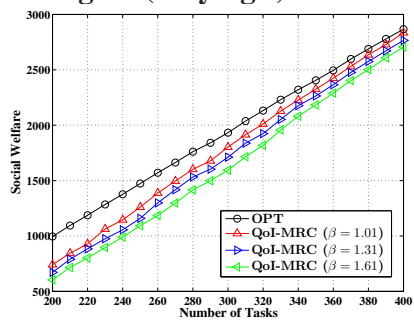

Figure 8: Social welfare for setting IV (varying $\beta$ )

\subsection{Simulation Results}

In Figure 3 and 4, we compare the social welfare generated by the QoI-VCG auction, the QoI-SRC auction and the MSW-Greedy auction. The social welfare of the QoI-VCG auction equals to the optimal solution of the SRC-WD problem. From Figure 3 and 4, we arrive at the conclusion that the social welfare of the QoI-SRC auction is close to optimal and far better than that of the baseline MSW-Greedy auction.

In Table 7 and 8, we show the comparison of the execution time of the QoI-VCG and QoI-SRC auctions. It is obvious from these two tables that the QoI-SRC auction executes in significantly less time than the QoI-VCG auction. With the increasing of the number of users and tasks, the execution time of the QoI-VCG auction gradually becomes so long that makes it infeasible to be utilized in practice. In contrast, the QoI-SRC auction keeps low execution time regardless of the growth of the user and task numbers. The QoI-SRC auction is much more computationally efficient than the QoI-VCG auction.

In Figure 5 and 6, we compare the social welfare generated by the QoI-MRC auction with the optimal social welfare in both set- 
ting III and IV. We fix the parameter $\beta=1.01$ and vary the choices of $\epsilon$. From the two figures, we observe that the QoI-MRC auction obtains close-to-optimal social welfare and it becomes closer to the optimal social welfare when $\epsilon$ approaches 0 .

\begin{tabular}{|c||c|c|c|c|c|c|c|c|}
\hline$N$ & 200 & 220 & 240 & 260 & 280 & 300 & 320 & 340 \\
\hline QoI-VCG & 10.19 & 16.06 & 11.22 & 11.71 & 58.64 & 63.14 & 79.37 & 10.51 \\
\hline QoI-SRC & 0.019 & 0.014 & 0.015 & 0.015 & 0.020 & 0.022 & 0.018 & 0.019 \\
\hline$N$ & 360 & 380 & 400 & 420 & 440 & 460 & 480 & 500 \\
\hline QoI-VCG & 43.52 & 93.44 & 94.25 & 273.6 & 52.54 & 72.26 & 860.9 & 2043 \\
\hline QoI-SRC & 0.019 & 0.021 & 0.021 & 0.019 & 0.023 & 0.021 & 0.021 & 0.024 \\
\hline
\end{tabular}

Table 7: Execution time (s) for setting I

\begin{tabular}{|c||c|c|c|c|c|c|c|c|}
\hline$M$ & 300 & 320 & 340 & 360 & 380 & 400 & 420 & 440 \\
\hline QoI-VCG & 18.70 & 1.337 & 2.715 & 15.47 & 21.42 & 43.38 & 88.57 & 224.3 \\
\hline QoI-SRC & 0.066 & 0.076 & 0.075 & 0.076 & 0.073 & 0.090 & 0.075 & 0.077 \\
\hline$M$ & 460 & 480 & 500 & 520 & 540 & 560 & 580 & 600 \\
\hline QoI-VCG & 67.85 & 50.68 & 183.5 & 229.3 & 474.8 & 751.1 & 1206 & 1269 \\
\hline QoI-SRC & 0.079 & 0.117 & 0.099 & 0.130 & 0.111 & 0.122 & 0.123 & 0.147 \\
\hline
\end{tabular}

Table 8: Execution time (s) for setting II

In Figure 7 and 8 , we fix the parameter $\epsilon=0.01$ and vary the choices of $\beta$. From these two figures, we also observe that the QoI-MRC auction obtains close-to-optimal social welfare and as $\beta$ approaches 1, it becomes closer to the optimal social welfare.

\section{CONCLUSION}

In this paper, we design QoI aware incentive mechanisms for MCS systems based on RC auctions. For the SRC auction, we design a truthful, individual rational and computationally efficient mechanism that approximately maximizes the social welfare with a guaranteed approximation ratio. For the MRC auction, we design an iterative descending mechanism that achieves close-to-optimal social welfare with a guaranteed approximation ratio while satisfying individual rationality and computational efficiency. Moreover, our theoretical analysis is validated through extensive simulations.

\section{References}

[1] X. Sheng, J. Tang, X. Xiao, and G. Xue, "Sensing as a service: Challenges, solutions and future directions," Sensors Journal, IEEE, 2013.

[2] "Medwatcher app," http://www.fda.gov/MedicalDevices/Safety/ ReportaProblem/ucm385880.htm.

[3] Y. Cheng, X. Li, Z. Li, S. Jiang, Y. Li, J. Jia, and X. Jiang, "Aircloud: A cloud-based air-quality monitoring system for everyone," in SenSys, 2014.

[4] D. Yang, G. Xue, X. Fang, and J. Tang, "Crowdsourcing to smartphones: Incentive mechanism design for mobile phone sensing," in Mobicom, 2012.

[5] L. Duan, T. Kubo, K. Sugiyama, J. Huang, T. Hasegawa, and J. Walrand, "Incentive mechanisms for smartphone collaboration in data acquisition and distributed computing," in INFOCOM, 2012.

[6] B. Faltings, J. Li, and R. Jurca, "Incentive mechanisms for community sensing," Computers, IEEE Transactions on, 2014.

[7] Z. Feng, Y. Zhu, Q. Zhang, L. Ni, and A. Vasilakos, "Trac: Truthful auction for location-aware collaborative sensing in mobile crowdsourcing," in INFOCOM, 2014.

[8] J.-S. Lee and B. Hoh, "Sell your experiences: a market mechanism based incentive for participatory sensing," in PerCom, 2010.

[9] Y. Zhang and M. van der Schaar, "Reputation-based incentive protocols in crowdsourcing applications," in INFOCOM, 2012.

[10] H. Xie and J. C. Lui, "Modeling crowdsourcing systems: Design and analysis of incentive mechanism and rating system," SIGMETRICS Perform. Eval. Rev., 2014.

[11] H. Xie, J. C. Lui, J. W. Jiang, and W. Chen, "Incentive mechanism and protocol design for crowdsourcing systems," in Allerton, 2014.

[12] Z. Feng, Y. Zhu, Q. Zhang, H. Zhu, J. Yu, J. Cao, and L. Ni, "Towards truthful mechanisms for mobile crowdsourcing with dynamic smartphones," in ICDCS, 2014.

[13] X. Zhang, Z. Yang, Z. Zhou, H. Cai, L. Chen, and X. Li, "Free market of crowdsourcing: Incentive mechanism design for mobile sensing," Parallel and Distributed Systems, IEEE Transactions on, 2014.
[14] D. Zhao, X.-Y. Li, and H. Ma, "How to crowdsource tasks truthfully without sacrificing utility: Online incentive mechanisms with budget constraint," in INFOCOM, 2014.

[15] S. He, D.-H. Shin, J. Zhang, and J. Chen, "Toward optimal allocation of location dependent tasks in crowdsensing," in INFOCOM, 2014.

[16] I. Koutsopoulos, "Optimal incentive-driven design of participatory sensing systems," in INFOCOM, 2013.

[17] T. Luo, H.-P. Tan, and L. Xia, "Profit-maximizing incentive for participatory sensing," in INFOCOM, 2014.

[18] Z. Song, C. Liu, J. Wu, J. Ma, and W. Wang, "Qoi-aware multitaskoriented dynamic participant selection with budget constraints," Vehicular Technology, IEEE Transactions on, 2014.

[19] E. H. Clarke, "Multipart pricing of public goods," Public Choice, 1971

[20] T. Groves, "Incentives in teams," Econometrica: Journal of the Econometric Society, 1973.

[21] J. Huang, R. A. Berry, and M. L. Honig, "Auction-based spectrum sharing," Mob. Netw. Appl., 2006.

[22] X. Chen and J. Huang, "Spatial spectrum access game: Nash equilibria and distributed learning," in MobiHoc, 2012.

[23] J. Jia, Q. Zhang, Q. Zhang, and M. Liu, "Revenue generation for truthful spectrum auction in dynamic spectrum access," in MobiHoc, 2009.

[24] H. Jin, G. Sun, X. Wang, and Q. Zhang, "Spectrum trading with insurance in cognitive radio networks," in INFOCOM, 2012.

[25] M. Dong, G. Sun, X. Wang, and Q. Zhang, "Combinatorial auction with time-frequency flexibility in cognitive radio networks," in INFOCOM, 2012.

[26] D. Yang, X. Fang, and G. Xue, "Truthful auction for cooperative communications," in MobiHoc, 2011.

[27] X. Chen, B. Proulx, X. Gong, and J. Zhang, "Social trust and social reciprocity based cooperative d2d communications," in MobiHoc, 2013.

[28] Q. Yu, J. Chen, Y. Fan, X. Shen, and Y. Sun, "Multi-channel assignment in wireless sensor networks: A game theoretic approach," in INFOCOM, 2010.

[29] M. Dong, H. Jin, G. Sun, X. Wang, W. Liu, and X. Wang, "Noncooperative game based social welfare maximizing bandwidth allocation in wsns," in GLOBECOM, 2011.

[30] Q. Zhang, Y. Wen, X. Tian, , X. Gan, and X. Wang, "Incentivize crowd labeling under budget constraint," in INFOCOM, 2015.

[31] Q. Li, Y. Li, J. Gao, B. Zhao, W. Fan, and J. Han, "Resolving conflicts in heterogeneous data by truth discovery and source reliability estimation," in SIGMOD, 2014.

[32] L. Su, Q. Li, S. Hu, S. Wang, J. Gao, H. Liu, T. Abdelzaher, J. Han, X. Liu, Y. Gao, and L. Kaplan, "Generalized decision aggregation in distributed sensing systems," in RTSS, 2014.

[33] Q. Li, Y. Li, J. Gao, L. Su, B. Zhao, M. Demirbas, W. Fan, and J. Han, "A confidence-aware approach for truth discovery on long-tail data," in Proceedings of the VLDB Endowment, 2014.

[34] S. Wang, D. Wang, L. Su, L. Kaplan, and T. F. Abdelzaher, "Towards cyber-physical systems in social spaces: The data reliability challenge," in RTSS, 2014.

[35] H. Li, B. Zhao, and A. Fuxman, "The wisdom of minority: Discovering and targeting the right group of workers for crowdsourcing," in $W W W, 2014$

[36] L. Blumrosen and N. Nisan, "Combinatorial auctions," Algorithmic Game Theory, 2007.

[37] M. Babaioff, R. Lavi, and E. Pavlov, "Single-value combinatorial auctions and algorithmic implementation in undominated strategies," $J$. ACM, 2009.

[38] M. J. Osborne and A. Rubinstein, A course in game theory. Cambridge, USA: The MIT Press, 1994, electronic edition.

[39] M. Babaioff, R. Lavi, and E. Pavlov, "Mechanism design for singlevalue domains," in AAAI, 2005.

[40] Z. Zheng, F. Wu, S. Tang, and G. Chen, "Unknown combinatorial auction mechanisms for heterogeneous spectrum redistribution," in $\mathrm{Mo}$ biHoc, 2014.

[41] R. B. Myerson, "Optimal auction design," Mathematics of operations research, 1981.

[42] S. Rajagopalan and V. Vazirani, "Primal-dual rnc approximation algorithms for (multi)-set (multi)-cover and covering integer programs," in FOCS, 1993.

[43] "Gurobi solver," http://www.gurobi.com/. 